\title{
An industry update: what is new in the field of therapeutic delivery?
}

\author{
Louise Rosenmayr-Templeton $*, 1$ \\ ${ }^{1}$ Tower Pharma Consulting, Auhofstrasse 197/10, 1130 Vienna, Austria \\ *Author for correspondence: louise.templeton@towerpharmacon.com
}

First draft submitted: 2 June 2017; Accepted for publication: 30 June 2017; Published online: 21 August 2017

Keywords: • competitor intelligence • intellectual property • partnering

This update covers the period 1-31 May 2017 and is based on information in press releases and company websites. During this month Thermo Fisher took the plunge into the pharmaceutical development and contract manufacturing market when it bought Patheon, Inc. Elsewhere deals included those between Durect and Sandoz, Sangamo and Pfizer, Exscientia and Sanofi and Protagonist and Janssen. Keytruda ${ }^{\circledR}$ became the first anticancer to receive US approval for any solid tumor with a specific biomarker, and Spherox, an articular cartilage product manufactured from a patient's own chondrocytes, looks set to gain EU approval after receiving a positive opinion from the relevant committees of the EMA.

\section{Mergers \& acquisitions}

\section{Thermo Fisher acquires Patheon}

On 15 May, Thermo Fisher Scientific, Inc. (MA, USA) entered the lucrative US $\$ 40$ billion pharmaceutical development and contract manufacturing market with its acquisition of Patheon, Inc. (NC, USA) [1]. Patheon is one of the world's largest pharmaceutical contractors for both small molecules and biopharmaceuticals, offering services for both drug substance and drug product from early development to commercialization. The deal involves the purchase of all of issued and outstanding shares of Patheon for $\$ 35.00$ per share in cash, resulting in a total purchase price of approximately $\$ 7.2$ billion assuming approximately $\$ 2.0$ billion of net debt. It is anticipated that the transaction will be complete by end 2017. The purchase of Patheon brings Thermo Fisher, who already provides a wide range of laboratory-based products, instruments and services to the healthcare industry, one step closer to its goal of becoming a one-stop shop for services to pharma and biotech companies.

\section{Collaborations}

\section{Durect sign agreement with Sandoz for Posimer ${ }^{\circledR}$}

On 8 May 2017 the Durect Corporation (CA, USA) made public that they had signed a development and commercialization agreement with Sandoz AG, a division of Novartis, specializing in generics and biosimilars, for Durect's POSIMIR ${ }^{\circledR}$ product in the USA [2]. POSIMIR contains the local anesthetic, bupivacaine, and employs the company's proprietary SABER ${ }^{\mathrm{TM}}$ technology. It has been designed to be instilled at surgical sites or injected into specific anatomic spaces under endoscopic guidance to deliver constant relief from pain over $72 \mathrm{~h}$ due to bupivacaine's local anaesthetic properties. The product is a semiviscous solution of the biodegradable esterified sugar, sucrose acetate isobutyrate, also containing $660 \mathrm{mg}$ bupivacaine base and benzyl alcohol. It forms a viscous depot following instillation at the surgical site to release bupivacaine at approximately $10 \mathrm{mg} / \mathrm{h}$ over $72 \mathrm{~h}$ [3].

According to the press release [2], the agreement involves Sandoz paying Durect $\$ 20$ million upfront, with the Californian company having the possibility to earn an additional $\$ 43$ million if it meets development and regulatory milestones. The deal also foresees up to $\$ 230$ million in sales related milestone payments if achieved, 
together with tiered double digit royalties on US product sales. The product is currently being tested in the PERSIST Phase III study which compares POSIMIR to bupivacaine $\mathrm{HCl}$ in patients undergoing gall bladder surgery. The transaction is planned to close in the second quarter of 2017.

\section{Senseonics \& TypeZero collaborate on artificial pancreas \& decision support systems}

Senseonics Holdings, Inc. (MD, USA), the developer of Eversense ${ }^{\circledR}$, an implantable continuous glucose monitoring system, has teamed up with TypeZero Technologies, Inc. (VA, USA), a personalized diabetes management company, to develop artificial pancreas and decision support systems that use the Eversense CGM System [4]. Eversense is made up of a small sensor based on fluorescence sensing technology inserted subcutaneously, a smart transmitter and mobile application, with the data being transmitted wirelessly to a mobile device.

The nonexclusive research and development license agreement relates to the integration of glucose readings from the Eversense CGM System into TypeZero's inControl software containing decision support and artificial pancreas system solutions. The aim is to use the readings from the Eversense sensor to develop TypeZero's artificial pancreas algorithms to adjust automatically and regulate insulin delivery via an insulin pump. It is also hoped that the data will enable the software decision support system to recommend optimal basal and bolus doses for patients using insulin pens.

\section{Nektar \& Takada to work together on cancer combination therapy}

Nektar Therapeutics (CA, USA) announced on 22 May 2017 that it had signed a research agreement with the Takeda Pharmaceutical Company Ltd (Osaka, Japan) through Takeda's subsidiary, Millennium Pharmaceuticals, Inc. (MA, USA) [5]. The purpose of the collaboration is to evaluate Nektar's NKTR-214, a CD122-biased agonist, in combination with five compounds from Takeda's anticancer portfolio, each with a different mechanism of tumor targeting. The compounds selected from the Takeda portfolio include a spleen tyrosine kinase inhibitor and a proteasome inhibitor. The combinations will be assessed in preclinical models of lymphoma, melanoma and colorectal cancer. The collaboration will involve each company providing the compounds for testing in the preclinical studies and sharing the study costs. However, both will keep the commercial rights to their respective compounds.

NKTR-214 is a PEGylated prodrug of the immunostimulant, recombinant human IL-2 [6]. The IL-2 core is conjugated to six polyethylene glycol chains. In vivo, the conjugate breaks down to release active IL-2 conjugates slowly. The investigational therapeutic agent is designed to overcome the issues associated with current recombinant human IL-2 therapy such as high frequency of dosing and dose-limiting side effects. NKTR-214 binds to CD122-specific receptors found on $\mathrm{CD} 8^{+}$effector $\mathrm{T}$ cells and natural killer cells. Binding stimulates a proliferation of these cells and their mobilization into the tumor microenvironment. It also increases expression of programmed death 1 (PD-1) receptors on their surface which, in turn, promotes the recognition of cancer cells by the immune system. In preclinical studies, NKTR-214 treatment resulted in an average ratio of 450:1 of $\mathrm{CD}^{+}$effector $\mathrm{T}$ cells, promoting tumor destruction, within the tumor compared to $\mathrm{CD} 4^{+}$regulatory $\mathrm{T}$ cells, which can suppress tumor-cell eradication [7]. NKTR-214 is being evaluated both as a single treatment and in combination with several investigational compounds including Takeda's.

\section{Exscientia enters multiple product deal with Sanofi}

A drug discovery company celebrating in May was Exscientia Ltd (Dundee, UK). Exscientia specializes in artificial intelligence-driven drug discovery for single target molecules, bispecifics and phenotypic drugs. On 9 May 2017 it signed a strategic research collaboration, and licence option agreement with Sanofi (Paris, France) for research into bispecific molecules suitable for the treatment of metabolic disease [8]. Progress in this area, for example, in diabetes, is currently hampered by a lack of single targets that can be successfully exploited for therapy. The two companies hope to overcome this hurdle by applying Exscientia's artificial intelligence and enabled automated design drug discovery platform to identify and validate combinations of drug targets efficiently which could work together synergistically. These targets selected would then become the focus of drug discovery using Exscientia's bispecific-small-molecule, lead-finding platform to validate the target pair further and generate potential candidates to be taken forward to full drug candidates by Sanofi provided they meet all quality criteria.

During the collaboration Exscientia will conduct all compound design, while Sanofi will carry out chemical synthesis. Sanofi will also be responsible for further assays, preclinical studies and any clinical trials conducted on compounds for which it has exercised its license option. Under the terms of agreement, Sanofi will 
provide research funding to identify the optimal target pairs followed by further remuneration for candidate prioritization. Candidates selected for development will be associated with preclinical and clinical milestone payments plus sales payments for those reaching the market with the total amount potentially payable by Sanofi to Exscientia being $€ 250$ million.

\section{Sangamo \& Pfizer sign agreement on hemophilia A gene therapy}

It was a month of good news for Sangamo Therapeutics, Inc. (CA, USA). On 10 May 2017 it made public that it had signed an exclusive, global collaboration and license agreement for the development and commercialization of gene therapy programs for hemophilia A with Pfizer (NY, USA) [9]. The agreement includes one of its four lead candidates, SB-525, which is soon to be tested in a Phase I/II trial. This was followed on 16 May by the news that the US FDA had granted Fast Track status to SB-525, which will facilitate its development and expedite its regulatory review [10]. The product already has an Orphan Drug designation.

Hemophilia $\mathrm{A}$ is a genetic disease caused by a mutation in the $F 8$ gene which results in a deficiency of Factor VIII, a blood clotting protein. It is estimated to affect 16,000 US patients and more than 150,000 globally. SB-525 is designed to be a one-off treatment by infusion. It consists of a recombinant adeno-associated virus (rAAV) vector, a Factor VIII gene construct and a proprietary, liver-specific promoter. It is hoped that SB-525 will enable continuous, therapeutic expression of Factor VIII protein in humans.

The agreement foresees Sangamo conducting the SB-525 Phase I/II study and certain manufacturing operations, with Pfizer being in charge of research, development, manufacturing and commercialization activities for SB-525 and any other products arising from the collaboration. Sangamo will also work with Pfizer on activities using viral delivery vectors. The deal involves Sangamo receiving a $\$ 70$ million upfront payment from Pfizer with the potential for the company to earn up to a further $\$ 475$ million in milestone payments plus tiered double-digit royalties on net sales. The $\$ 475$ million figure is split into $\$ 300$ million for the commercialization and development of SB-525 and up to $\$ 175$ million for any other hemophilia A gene therapy product candidates that may arise out of the collaboration. If SB-525 is successful in the clinic and obtains regulatory approval, it will be the first gene therapy product approved in the USA and has the potential to revolutionize the treatment of hemophilia A.

\section{Milestone news}

\section{Heptares earns milestone payment from Teva}

May 2017 brought good news for Heptares Therapeutics (London, UK), a subsidiary of the Sosei Group Corporation (Tokyo, Japan) focused on discovering and developing medicines targeting $\mathrm{G}$ protein-coupled receptors (GPCRs). Teva Pharmaceutical Industries Ltd (Jerusalem, Israel), its partner since 2015, has selected a small molecule calcitonin gene-related peptide antagonist (CGRP) for further preclinical studies [11]. The candidate is being investigated for its potential as a treatment for migraine, a condition affecting 36 million people in the USA alone. CGRP, which is found in nerve cells in the trigeminal ganglion, acts through GPCRs and its presence is associated with migraine with its levels being raised during attacks. Moreover, the peptide has been shown to trigger migraine episodes in patients and blocking its action has been demonstrated to ameliorate pain and prevent migraine.

The candidate was discovered by Heptares in partnership with Teva using its structure-based drug design platform [12]. The foundation of this platform is the $S t a R^{\circledast}$ protein technology which involves the insertion of point mutations to a target GPCR to increase thermostability without compromising its pharmacology and, thus, enabling the production of stable purified conformations of the receptor which can be used for drug candidate bindings studies.

The candidate selection represents the first milestone in the licensing and drug discovery agreement between the two companies, which gives Teva exclusive rights to develop, manufacture and commercialize CGRP antagonists resulting from the partnership for the treatment of migraine. The selection triggered a $\$ 5$ million payment.

\section{Product approvals}

\section{First cancer treatment to be approved for any solid tumor with a specific biomarker}

On 23 May 2017 the US FDA gave accelerated approval to Keytruda ${ }^{\circledR}$ (pembrolizumab) from Merck \& Co (NJ, USA) for the treatment of adult and children with unresectable or metastatic solid tumors exhibiting the biomarker known as microsatellite instability-high (MSI-H) or mismatch repair deficient (dMMR) [13]. The FDA 
approved the product based on tumor response rate data including the duration of the response [14].

This represents the first time a cancer treatment has been approved for the tumors with a common biomarker as opposed to the location of the primary tumor. Tumors bearing MSI-H and dMMR biomarkers have defective DNA repair mechanisms and are most frequently found in colorectal, endometrial and gastrointestinal cancers, but can also be present in other types of cancer.

Keytruda is a humanized antibody which binds to the PD-1 receptor and is already on the market for the treatment of certain patients with metastatic non-small-cell lung cancer, metastatic melanoma, recurrent or metastatic head and neck cancer, urothelial carcinoma and refractory classical Hodgkin lymphoma. Postapproval Merck is required to confirm the clinical benefits of Keytruda in additional patients with MSI-H or dMMR tumors in order to ensure that the product retains the expanded indication. These studies are currently ongoing.

\section{CO.DON receives positive opinion from EMA}

CO.DON AG (Berlin, Germany) received good news this month from the EMA on Spherox, its articular cartilage product $[15,16]$. The product is indicated for the treatment of symptomatic articular cartilage defects in the femoral condyle (the ball-shaped end of the thigh bone in the knee) and the knee cap provided the defects are not more than $10 \mathrm{~cm}^{2}$ in size. In this treatment healthy cartilage is removed from the patient and used to grow chrondrocyte spheroids (spherical aggregates of chondrocytes) in CO.Don's sterile facilities. These are then inserted into the patient's knee where they form new tissue.

The recommendation was based on the results of two clinical trials, a Phase II involving 75 patients with defects which ranged in size from 4 to $10 \mathrm{~cm}^{2}$ and a Phase III with 102 patients presenting with defects of $1-4 \mathrm{~cm}^{2}$. The trials showed that use of Spherox was associated with a statistically significant improvement in the scores achieved using a Knee injury and Osteoarthritis Outcome Score questionnaire which evaluated aspects such as pain, impact on daily living, sport and recreational activities and quality of life from the patients' perspective.

CO.DON has committed to follow-up patients on the ongoing trials for a further 60 months in order to assess the long-term impact of therapy. The product has already been on the German market for a number of years and around 11,000 patients have already been successfully treated.

\section{Clinical trials}

\section{Positive Phase III results for buprenorphine depot, CAM2038}

Early in the month Braeburn Pharmaceuticals Inc. (NJ, USA) and Camurus AB (Lund, Sweden) announced positive top-line Phase III results in a long-term safety study of their weekly and monthly buprenorphine depot injection, CAM2038, for patients with moderate-to-severe opioid use disorder [17]. This is the second of two Phase III trials to demonstrate the safety and efficacy of CAM2038. Buprenorphine is the current therapy of choice for this condition which is said to affect more than 2.6 million people in the USA alone with over 1 million patients being treated with buprenorphine in the USA and Europe. Currently, buprenorphine is mostly administered sublingually in daily doses. It is a $\mathrm{m} \mu$-opioid receptor partial agonist which binds only partially to the $\mathrm{m} \mu$-opioid receptor and, hence, antagonizes the effect of full agonist opioids administered at the same time. Both the weekly and monthly depot injections have been developed in multiple doses allowing the dose to be tailored to the patient's needs. The depot injections have been formulated using Camurus' Fluid-Crystal ${ }^{\circledR}$ technology, which involves the formation of a liquid crystalline gel at the depot site following administration of the dissolved drug in a lipid-based liquid to entrap and slowly release the buprenorphine [18].

The Phase III study was conducted as an open-label multicenter trial with a treatment phase of 48 weeks and assessed the safety of CAM2038 administered once weekly and once monthly in adult outpatients with opioid use disorder. No opioid overdoses were reported for patients in the trial and the main side effect (20\% of patients) was injection site-reactions. These were generally mild $(16.3 \%)$ or moderate $(3.5 \%)$ with only one patient reporting severe pain. Other common side effects were headache, nausea, vomiting, nasopharyngitis and urinary tract infection (in each case reported by $<10 \%$ of patients). Efficacy was evaluated using weekly and monthly urine toxicology tests which on average showed that $75 \%$ of the urine samples were negative for illicit opioids during the treatment period.

\section{Inotersen meets Phase III end points}

Ionis Pharmaceuticals, Inc. (CA, USA) released positive data from the Phase III NEURO-TTR study of inotersen (IONIS-TTRRx) in patients with familial amyloid polyneuropathy (FAP) which showed the product met 
both primary endpoints [19]. FAP is a rare genetic disease which results in the production of mutant transthyretin (TTR), whose wild-type has the important role of carrying thyroxine and retinol in the body. In FAP the wildtype and mutant TTR misfold and accumulate as amyloid fibrils in tissues and this leads to nerve damage, loss of motor function and eventually death. Inotersen (IONIS-TTRRx) is a generation 2.0+ antisense drug which inhibits production of both the mutant and wild-type forms of TTR protein and could potentially be a treatment for all types of TTR amyloidosis including FAP [20]. Globally it is thought that around 10,000 patients are currently suffering from FAP.

The randomized double-blind, placebo-controlled Phase III trial involved 172 patients with FAP over 15 months. Efficacy measures were the impact of inotersen on neurological dysfunction and on quality-of-life using a modified Neuropathy Impairment Score $+7(\mathrm{mNIS}+7)$ and the Norfolk Quality of Life Questionnaire-Diabetic Neuropathy (Norfolk QOL-DN) total score. Both assessment methods gave statistically significant better scores for patients treated with inotersen compared with those receiving placebo. However, three serious adverse cases of thrombocytopenia were observed in inotersen-treated patients including one fatality. Long-term safety and efficacy data with inotersen are currently being collected in an open-label extension of the Phase III NEURO-TTR study in which $95 \%$ of those who completed the trials have entered the open-label extension.

\section{ViaCyte set to start trial of PEC-direct cell therapy in Type 1 diabetes}

On 22 May 2017 came the news from ViaCyte, Inc. (CA, USA) that both the FDA and Health Canada had accepted the company's application to start a trial to evaluate its PEC-Direct ${ }^{\mathrm{TM}}$ product. PEC-Direct is an islet cell replacement therapy which is being developed as a potential cure for Type 1 diabetic patients with a high risk of developing acute life-threatening complications [21]. The stem-cell therapy is foreseen as a replacement to islet transplants in Type 1 patients who have hypoglycemia unawareness, extreme glycemic lability, and/or severe hypoglycemic episodes. It is estimated that 140,000 people in the USA and Canada could benefit from such a treatment. The planned open-label trial in 40 patients will assess safety and evidence of efficacy. The primary efficacy end point in the study will be insulin production at clinically relevant levels using C-peptide as a biomarker.

The PEC-Direct consists of PEC-01 ${ }^{\mathrm{TM}}$ cells, which are stem cell-derived pancreatic progenitor cells, in an open device in which direct vascularization of the cells can take place. It is hoped that following implantation the cells will differentiate and mature to form the cell types found in healthy pancreatic tissue including insulinproducing $\beta$ cells. Unlike islet transplants the device and the implanted cells, which are anticipated to remain in or close to it, can be removed if necessary and treatment terminated should issues occur. Following implantation immune suppression therapy will be necessary as the open device does not afford protection from immune cell attack.

\section{Conference news}

\section{Arymo $^{\circledR}$ ER harm reduction modeling analysis}

The Egalet Corporation (PA, USA) presented data from a harm reduction model for its sustained release morphine sulfate product, Arymo ${ }^{\circledR}$ ER, at the 36th American Pain Society annual meeting on 18 May 2017 [22]. Arymo ER was approved by the US FDA at the start of the year [23] and is formulated in three dosage strengths: 15,30 and $60 \mathrm{mg}$ in the company's proprietary Guardian ${ }^{\mathrm{TM}}$ technology. This technology is based on the injection molding of tablets containing polyethylene oxide to form with a hard matrix with both extended release and abuse-deterrent properties.

The model includes data from diagnosed abusers and information on Arymo ER's physical and chemical properties based on its in vitro Category 1 abuse-deterrent data. The model predicted that Arymo ER may be associated with fewer abuse-related events such as emergency department visits, treatment in out-patient clinics, hospital admissions, injection-related diseases and deaths. The true extent of its effectiveness in hindering abuse will, of course, only be known once the product has been marketed for some time. The modeling will help support Egalet's market launch activities (it was launched in the USA at the end of March) [24]. 


\section{Patents}

Key patent issued for protagonist's oral peptide IL-23 receptor antagonists

Protagonist Therapeutics, Inc. (CA, USA) announced on 4 May 2017 that a key US patent had been issued covering the company's orally stable peptide antagonists of the IL-23 receptor (IL-23R), including its clinical candidate, PTG-200 [25,26]. The patent provides composition of matter protection until 2035 for PTG-200 and covers the use of the oral peptide inhibitors of IL-23R to treat inflammatory bowel disease (IBD). The PTG-200 is intended as an oral gastrointestinal-restricted treatment for IBD including Crohn's Disease which will provide an alternative to the TNF- $\alpha$ antibody drugs such as Humira ${ }^{\circledR}$ and Remicade ${ }^{\circledR}$ currently approved for moderateto-severe IBD. These can only be administered by injection.

The month of May also ended well for the company with the news that they had signed a global agreement with Janssen Biotech, Inc. (NJ, USA) to co-develop and commercialize PTG-200 for IBD in a deal worth \$50 million upfront plus, if achieved, up to $\$ 940$ million associated with development and sales milestones together with double-digit tiered royalties on net sales [27]. Phase I clinical trials on PTG-200 are planned in the second half of 2017.

\section{Ocugenexpands patent portfolio}

Ocugen, Inc. (PA, USA) continued to build its world-wide patent protection this month for its treatment, OCU200, for wet age-related macular degeneration and other ophthalmic neovascular diseases [28]. The company received a Notification of Intent to Grant Patent from the European Patent Office for a patent covering composition of matter and methods of use for OCU200. This adds to those already issued by the United States Patent and Trademark Office (US Patent No. 9290562) and the Japan Patent Office (JPO Patent Publication No. 6073888). OCU200 is a fusion protein which has the antiangiogenic properties of tumstatin, an endogenous fragment of collagen IV, and transport properties of transferrin. Tumstatin downregulates angiogenesis in active endothelial cells involved in choroidal neovascularization by binding to $\alpha \mathrm{V} \beta 3$ integrins. This results in regression of endothelial cells. Transferrin and its receptor are highly expressed within the retina and the iron-binding protein has been shown to facilitate transport of macromolecules into retinal pigment epithelial cells. OCU200 is currently in preclinical testing with a Phase I study planned for 2018 [29].

\section{Financial \& competing interests disclosure}

The author has no relevant affiliations or financial involvement with any organization or entity with a financial interest in or financial conflict with the subject matter or materials discussed in the manuscript. This includes employment, consultancies, honoraria, stock ownership or options, expert testimony, grants or patents received or pending, or royalties.

No writing assistance was utilized in the production of this manuscript.

\section{References}

1 Thermo Fisher Scientific to Acquire Patheon, a Leading Contract Development and Manufacturing Organization (CDMO). http://ir.thermofisher.com/investors/news-and-events/news-releases/news-release-details/2017/Thermo-Fisher-Scientific-toAcquire-Patheon-a-Leading-Contract-Development-and-Manufacturing-Organization-CDMO/default.aspx

2 DURECT and Sandoz Have Signed a $\$ 293$ Million Development and Commercialization Agreement for POSIMIR ${ }^{\circledR}\left(\right.$ SABER $^{\circledR}$ Bupivacaine) Covering the United States. http://investors.durect.com/phoenix.zhtml?c=121590\&p=irol-newsArticle \&ID=2270681

3 Doraiswamy A, Meisner J, Verity N. A new method for treating postoperative pain associated with laparoscopic surgery. Poster session presented at: 2016 Society of American Gastrointestinal and Endoscopic Surgeons Annual Meeting. Boston, MA, USA, 16-19 March 2016.

www.durect.com/science-technologies/publications/posimir-saber-bupivacaine/

4 Senseonics and TypeZero Sign Development Agreement for Artificial Pancreas and Decision Support Systems. www.senseonics.com/investor-relations/news-releases/2017/05-11-2017-215934769

5 New Research Collaboration Between Nektar and Takeda to Explore Combination Cancer Therapy Approaches with NKTR-214, a CD122-Biased Agonist, and Five Takeda Cancer Therapy Compounds. http://ir.nektar.com/releasedetail.cfm?ReleaseID=1027335

6 Nektar Therapeutics. www.nektar.com/science/research-platform-hidden

7 Charych DH, Hoch U, Langowski JL et al. NKTR-214, an engineered cytokine with biased il2 receptor binding, increased tumor 
exposure, and marked efficacy in mouse tumor models. Clin Cancer Res. 22(3), 680-690 (2016).

\section{Sanofi.}

www.exscientia.co.uk/news

9 Sangamo Therapeutics and Pfizer Announce Collaboration for Hemophilia A Gene Therapy.

http://investor.sangamo.com/press-releases/detail/359/sangamo-therapeutics-and-pfizer-announce-collaboration-for

10

http://investor.sangamo.com/press-releases/detail/363/sangamo-receives-fast-track-designation-from-the-fda-for

11 Heptares to Receive US\$5 Million Milestone Payment from Teva.

www.heptares.com/news/304/74/Heptares-to-Receive-US-5-Million-Milestone-Payment-from-Teva.html

12 Heptares Therapeutics.

www.heptares.com

13 FDA Approves First Cancer Treatment for Any Solid Tumor with a Specific Genetic Feature.

www.fda.gov/newsevents/newsroom/pressannouncements/ucm560167.htm

14 FDA Approves Merck’s KEYTRUDA ${ }^{\circledR}$ (Pembrolizumab) for Adult and Pediatric Patients with Unresectable or Metastatic,

Microsatellite Instability-High (MSI-H) or Mismatch Repair Deficient (dMMR) Tumors.

www.mrknewsroom.com/news-release/prescription-medicine-news/fda-approves-mercks-keytruda-pembrolizumab-adult-andpediatr

15 CO.DON: Decisive Step Towards European Expansion.

www.codon.de/news-overview/press-release/read-news/article/codon_entscheidender_meilenstein_schritt_auf_dem_weg_der_ europaeischen_expansion.html? L=1\&cHash=f8ddffbcdc15cccc7e2edee 9 b777375e

16 Summary of CHMP Opinion: Spherox.

www.ema.europa.eu/docs/en_GB/document_library/Summary_of_opinion_-_Initial_authorisation/human/002736/

WC500228068.pdf

17 Braeburn Pharmaceuticals and Camurus Announce Positive Top-Line Results from Long-Term Phase III Safety Study of CAM2038.

www.prnewswire.com/news-releases/braeburn-pharmaceuticals-and-camurus-announce-positive-top-line-results-from-long-termphase-3-safety-study-of-cam2038-300449203.html

Camurus. www.camurus.com/camurus

19 Ionis Pharmaceuticals Announces Phase III NEURO-TTR Study of Inotersen (IONIS-TTR Rx) Meets Both Primary End points. www.prnewswire.com/news-releases/ionis-pharmaceuticals-announces-phase-3-neuro-ttr-study-of-inotersen-ionis-ttr-rx-meetsboth-primary-endpoints-300457281.html

20 Ionis Pharmaceutical.

www.ionispharma.com/pipeline

21 ViaCyte Receives IND Allowance from FDA and Clearance from Health Canada to Commence International Clinical Trial of PEC-Direct Cell Therapy for High-Risk Type 1 Diabetes.

www.prnewswire.com/news-releases/viacyte-receives-ind-allowance-from-fda-and-clearance-from-health-canada-to-commenceinternational-clinical-trial-of-pec-direct-cell-therapy-for-high-risk-type-1-diabetes-300461166.html

22 Egalet Announces Results of Arymo ${ }^{\circledR}$ ER Harm Reduction Modeling Analysis. http://egalet.investorroom.com/2017-05-18-Egalet-Announces-Results-of-ARYMO-R-ER-Harm-Reduction-Modeling-Analysis

23 Egalet Receives FDA Approval for ARYMO ${ }^{\mathrm{TM}}$ ER (Morphine Sulfate) C-II, an Extended-Release Morphine Product Formulated with Abuse-Deterrent Properties for Treatment of Chronic Pain. Press Release Egalet Corporation website.

http://egalet.investorroom.com/news-releases?year=2017

24 Egalet Corporation. http://egalet.com

25 Key Patent Issues Covering Protagonist Therapeutics' Oral Peptide IL-23 Receptor Antagonists, including the Development Candidate PTG-200.

www.prnewswire.com/news-releases/key-patent-issues-covering-protagonist-therapeutics-oral-peptide-il-23-receptor-antagonistsincluding-the-development-candidate-ptg-200-300452029.html

26 Protagonist Therapeutics, Inc. US9624268 (2017).

27 Protagonist Therapeutics Enters into Worldwide Agreement with Janssen to Co-Develop and Commercialize PTG-200 for Inflammatory Bowel Disease.

http://investors.protagonist-inc.com/phoenix.zhtml?c=254413\&p=irol-newsArticle \&ID = 2277187

28 Ocugen Expands Global Patent Portfolio for OCU200.

www.prnewswire.com/news-releases/ocugen-expands-global-patent-portfolio-for-ocu200-300453106.html

29 
